\section{Evaluation of a new cataract surgery referral pathway}

JC Park ${ }^{1}$, AH Ross ${ }^{2}$, DM Tole², JM Sparrow², $J$ Penny ${ }^{2}$ and MV Mundasad ${ }^{2}$

\begin{abstract}
Aims To compare the quality of referrals and listing rates of direct optometric referrals $v s$ traditional GP referrals for cataract surgery. Methods A retrospective cohort of 124 patients referred for cataract surgery was identified (62 via optometric pathway and 62 via GP pathway). The quality of the referral was assessed by establishing if it contained adequate information relating to the College of Optometrists' referral framework document. Age, sex, drug history, listing rate, operative rate, and visual acuity (best corrected) at referral and at the postoperative visit were recorded and compared between the two referral pathways using the Fisher's
\end{abstract} exact test.

Results Optometric referrals, relative to GP referrals, were more likely to include information relating to objective visual loss (100 vs $87 \%, P=0.0061$ ) and to counsel the patient (97 vs $\mathbf{1 8 \%}, \boldsymbol{P}=\mathbf{0 . 0 0 0 1 )}$ ). GP referrals, relative to optometric referrals, were more likely to comment on personal circumstances (32 vs 3\%, $P=0.0001$ ), past medical history (95 vs 68\%, $P=0.0001$ ), and drug history (94 vs 69\%, $P=0.0009$ ). Operative rates were higher for the optometric direct referrals relative to GP referrals (87 vs $69 \%, P=0.0284$ ). There was no difference in the visual acuity before or after surgery between the pathways.

Conclusions Optometric direct cataract referrals provide better information on objectively measured vision and better delivery of preoperative counselling. Traditional GP referrals contain better medical history, drug information, and details of personal circumstances. Rates of surgery were slightly higher with optometric referrals. Eye (2009) 23, 309-313; doi:10.1038/sj.eye.6703075; published online 8 February 2008

Keywords: cataract; referral; optometrists; patient pathway

\section{Introduction}

Cataract continues to be a major cause of visual impairment and can currently only be treated by surgery. Following the Government's commitment to streamline cataract referrals, ${ }^{1}$ a number of operational frameworks have been produced..$^{2-4}$ These frameworks aim to improve the co-management of cataract by optometrists, general practitioners (GPs), and ophthalmologists. This includes the shortening of the cataract patient pathway in an attempt to increase patient satisfaction, to extend the role of the optometrist, and to reduce workload for hospital eye services (HES) and GPs.

To achieve this aim a major change in practice has been introduced - the direct referral of patients with cataract from optometrists to ophthalmologists. These operational frameworks encourage shortening of the traditional patient journey from eight steps to five, with no need for the patient to see the GP. However, it remains vital for the GP to provide the medical history, with direct referral forms initially being filled out by the optometrist, and subsequently sent to their GP for completion. Drug history is of particular importance since anticoagulants, antiplatelet agents, and $\alpha$-blockers may have an impact on cataract surgery.

The direct referral documents highlight the importance of referring those patients who have cataract as the main cause of visual loss, which significantly affects their lifestyle. In addition, they state that only patients who remain keen for surgery after counselling should be referred, and if they are referred then information about the patient's condition (such as visual loss, medical history, and personal circumstances) must be included.

A small number of published reports ${ }^{5,6}$ are available that have compared the optometric direct pathway with the traditional GP pathway. These reports have assessed listing rates, operative outcomes, patient satisfaction, and
${ }^{1}$ West of England Eye Unit, Royal Devon and Exeter Hospital, Exeter, England, UK

${ }^{2}$ Bristol Eye Hospital, Bristol, England, UK

Correspondence: JC Park, West of England Eye Unit, Royal Devon and Exeter Hospital,

Barrack Road,

Wonford, Exeter, Devon EX2 5DW, UK

Tel: + 01392406008 ;

Fax: + 01392406022

E-mail: jonparkgfc@

hotmail.com

Received: 22 August 2007 Accepted in revised form: 24 November 2007 Published online: 8 February 2008

Proprietary interests/research funding/competing interests: None 
number of HES clinics saved, ${ }^{5,6}$ and their data supports the optometric direct cataract referral pathway.

A recent study assessed the quality of these referrals relative to the document produced in 2000 by the Department of Health, 'Action on Cataracts'. ${ }^{1}$ This report found that the operative rate was higher for referrals that included information relating not just to the presence of cataract, but also to the detrimental effect on lifestyle and the patient's willingness for surgery. ${ }^{7}$

Since this study, a number of more detailed operational frameworks have been produced by the Department of Health and the College of Optometrists, providing suggestions relating to the quality of cataract referrals. ${ }^{2-4}$

The aim of this study is to compare the quality of referrals and listing rates of direct optometric referrals vs traditional GP referrals for cataract surgery, with reference to these new pathways relating to the quality of cataract referrals.

\section{Materials and methods}

A retrospective cohort of patients referred for cataract surgery in Avon and South Gloucestershire was identified. This included patients whose referral was initiated by their GP (traditional GP pathway, using the General Ophthalmic Services 18 form) or by their optometrist (direct optometric pathway, using the direct referral form). Patient notes were obtained by consecutive case note selection for referrals made from March to May 2006 (14-17 months after the initiation of a new direct optometric referral pathway). A ratio of $1: 1$ (traditional referral/optometric referral) was maintained throughout the case note selection to minimise temporal bias.

The optometrists who referred patients directly had received training relating to the direct referral process, written information provision, and counselling in the form of a lecture and discussion session.

The required sample size of 62 patients per group was estimated based upon desired $80 \%$ power to demonstrate a statistically significant difference $(P<0.05)$ of more than $20 \%$ between the two referral pathways, ie, 70 vs $90 \%$.

Age and sex were recorded to establish fundamental demographics. The quality of each referral was then assessed, by establishing if the referral contained adequate information relating to each referral item suggested by the College of Optometrists' document, ${ }^{3}$ 2005 (Box 1).

Surprisingly, neither the College of Optometrists ${ }^{3}$ nor the NHS Executive ${ }^{1}$ suggest that the cataract referral should include a drug/allergy history, except for the NHS Executive ${ }^{1}$ suggestion of stating if the patient is on
Box 1 Information items for referral suggested by the College of Optometrists ${ }^{3}$ (2005), for inclusion in cataract referrals

(1) Objective loss of visual performance eg: visual acuity and/or reading speed and/or contrast sensitivity

(2) Subjective loss of visual performance eg: dim vision, glare, difficulty reading, or accomplishing everyday tasks, or in recognising faces or viewing the television

(3) Ocular comorbidity

(4) Increased anisometropia causing asthenopic symptoms

(5) Rapid decrease in visual acuity

(6) Patient's personal circumstances eg: immobility, deafness, living alone, caring for dependent

(7) Whether or not the patient has been counselled and remains keen for surgery

(8) Assessment of general health eg: blood pressure

(9) Presence or absence of acute blepharitis (a contraindication to surgery)

an anticoagulant. As allergies and drugs (such as anticoagulants, antiplatelet agents, and $\alpha$-blockers) can have a significant impact on cataract surgery, the authors decided that drug/allergy history was important to record.

The listing and operative rates were calculated for each referral pathway. For patients referred who did not proceed to surgery, the reasons for not being listed were identified.

The best-corrected visual acuity at referral and 4-8 weeks after surgery (provided by optometrist or cataract nurse practitioner) were recorded for each referral pathway. The average visual acuities were calculated by converting each individual visual acuity to its corresponding $\log M A R$ value ( $\log _{10}$ inverse Snellen's fraction), obtaining the mean, and then converting this back to a Snellen's fraction.

It was anticipated that the electronic patient record database would be a useful tool in obtaining data. However, at the time of the study, not all the information desired for this study was available from the electronic patient record database. For example, it was not possible for community optometrists located remote from the trust to enter data (such as visual acuity). Optometrists would, therefore, send information by post to hospital for filing in the patient notes. It was, therefore, necessary to go through each set of patient notes to obtain all the required information.

Group differences between modes of referral were assessed for statistical significance $(P<0.05)$ using the Fisher's exact test. The Mann-Whitney U-test was used to establish if the differences between the two referral pathways were statistically significant for visual acuities. 


\section{Results}

The patient notes of 124 patients referred for cataract surgery (62 via optometric pathway and 62 via traditional GP pathway) were identified by consecutive case note selection for referrals made from March to May 2006. For the 62 patients referred via the optometric pathway the mean age was 78 years (range 50-95 years), with sex ratio approximately 1:2 (23 men, 39 women). For the 62 patients referred via the GP pathway, the mean age was 76 years (range 35-95 years), with sex ratio approximately 1:2 (20 men, 42 women).

There was no statistically significant difference in average, best-corrected visual acuities presurgery
(6/15 for GP pathway, 6/18 for optometric pathway) or post surgery (6/8 for both pathways).

Table 1 shows the percentage of referrals that respected each of the data items (as suggested by the College of Optometrists $^{3}$ ) and also drug history for both referral pathways. Table 2 shows the statistical subset analysis for markers of subjective visual loss for both pathways.

Table 3 displays the listing and operative rates for both pathways.

Statistical subset analysis for markers of objective visual loss revealed that all referrers (both GPs and optometrists) report only visual acuity (nobody reported contrast sensitivity or reading speed).

Table 1 Percentage of referrals that respected each of the College of Optometrists ${ }^{3}$ suggested items and also drug history

\begin{tabular}{|c|c|c|c|c|c|}
\hline \multirow[t]{2}{*}{ Referral information items } & \multicolumn{2}{|c|}{$\begin{array}{c}\text { Referrals for traditional GP pathway that included } \\
\text { referral item }\end{array}$} & \multicolumn{2}{|c|}{$\begin{array}{c}\text { Referrals for optometric direct pathway } \\
\text { that included referral item }\end{array}$} & \multirow[t]{2}{*}{ P-value } \\
\hline & Number & $\%$ & Number & $\%$ & \\
\hline Objective visual loss & 54 & $87(76-93)$ & 62 & 100 & 0.0061 \\
\hline Subjective visual loss & 48 & 77 (64-85) & 46 & $74(62-84)$ & 0.8342 \\
\hline Anisometropia & 1 & $2(0-11)$ & 0 & 0 & 1.0000 \\
\hline Rapid fall in visual acuity & 0 & 0 & 0 & 0 & NA \\
\hline Personal circumstances & 20 & $32(22-45)$ & 2 & $3(1-12)$ & $<0.0001$ \\
\hline Received counselling & 11 & $18(10-30)$ & 60 & 97 (88-99) & $<0.0001$ \\
\hline Past medical history & 59 & $95(86-98)$ & 42 & $68(55-78)$ & 0.0001 \\
\hline Acute blepharitis & 0 & 0 & 0 & 0 & NA \\
\hline Ocular comorbidities & 62 & 100 & 62 & 100 & NA \\
\hline Drug history & 58 & $94(84-98)$ & 43 & $69(57-80)$ & 0.0009 \\
\hline
\end{tabular}

Abbreviations: GP = general practitioner; $\mathrm{NA}=$ not applicable.

$P$-value (Fisher's exact test). 95\% confidence intervals (in parentheses for percentage estimate).

Table 2 Markers of subjective visual loss, which were given on the referral form

\begin{tabular}{|c|c|c|c|c|c|}
\hline \multirow[t]{2}{*}{ Components of subjective visual loss } & \multicolumn{2}{|c|}{$\begin{array}{l}\text { Referrals for traditional GP pathway } \\
\text { that included referral item }\end{array}$} & \multicolumn{2}{|c|}{$\begin{array}{l}\text { Referrals for optometric direct pathway } \\
\text { that included referral item }\end{array}$} & \multirow[t]{2}{*}{ P-value } \\
\hline & Number & $\%$ & Number & $\%$ & \\
\hline Visual loss/dim vision/blurring & 45 & $73(60-82)$ & 1 & $2(0-11)$ & $<0.0001$ \\
\hline Difficulty reading & 9 & $15(8-26)$ & 0 & 0 & 0.0029 \\
\hline Difficulty seeing TV/faces & 2 & $5(2-14)$ & 0 & 0 & 1.0000 \\
\hline Glare & 1 & $2(0-11)$ & 1 & $2(0-11)$ & 1.0000 \\
\hline Difficulty driving & 6 & $10(4-20)$ & 3 & $5(2-14)$ & 1.0000 \\
\hline
\end{tabular}

Abbreviation: GP = general practitioner.

$P$-value (Fisher's exact test). 95\% confidence intervals (in parentheses for percentage estimate).

Table 3 Listing and operative rates for both referral pathways

\begin{tabular}{|c|c|c|c|c|c|c|c|}
\hline \multirow[t]{2}{*}{ Rates } & \multicolumn{2}{|c|}{ Traditional GP pathway } & \multicolumn{2}{|c|}{ Optometric direct pathway } & \multirow[t]{2}{*}{ P-value } & \multirow[t]{2}{*}{ Odds ratio } & \multirow[t]{2}{*}{$C I$} \\
\hline & Number & $\%$ & Number & $\%$ & & & \\
\hline Listing rate & 43 & 69 & 55 & 88 & 0.0142 & 3.47 & $1.34-9.02$ \\
\hline Operative rate & 43 & 69 & 54 & 87 & 0.0284 & 2.98 & $1.19-7.47$ \\
\hline
\end{tabular}

Abbreviations: $\mathrm{GP}=$ general practitioner; $\mathrm{CI}=95 \%$ interval for odds ratio.

$P$-value (Fisher's exact test). 
More patients from the traditional GP pathway were not listed, because the cataract was found to have no effect on their lifestyle (12 from GP pathway, 4 from direct pathway), or because the patient declined surgery (four from GP pathway, two from direct pathway), or for other reasons (three from GP pathway, two from direct pathway). However, these differences in individual reasons why patients were not listed for surgery did not reach statistical significance.

\section{Discussion}

The results from this study allow the successful review of the study's aim, as the sample size is sufficient and the basic demographics of the study sample for each referral pathway were similar.

There are some important differences in referral quality between the direct optometric pathway and the traditional GP pathway. Optometric referrals, relative to GP referrals, are more likely to include data relating to objective visual loss (100 vs 87\%, $P=0.0061$ ) and more likely to counsel the patient (97 vs 18\%, $P=0.0001$ ). However, GP referrals, relative to optometric referrals, are more likely to comment on personal circumstances (32 vs 3\%, $P=0.0001$ ), past medical history (95 vs 68\%, $P=0.0001$ ), and drug history (94 vs 69\%, $P=0.0009$ ). The main reason why referrals initiated by the optometrist are more likely to include data relating to objective visual loss is probably due to the fact that recording of visual acuity is routinely practised by optometrists, and the main bulk of the direct cataract referral form relates to the refractive prescription. Fortunately, regardless of referral pathway, the majority of referrals do report visual acuity (100\% via optometric pathway, $87 \%$ via GP pathway, $P=0.0061)$. It is interesting to note that the College of Optometrists recommend that objective visual loss can be expressed by visual acuity, reading speed, or contrast sensitivity. Not a single referrer (GP or optometrist) commented on reading speed or contrast sensitivity, suggesting that visual acuity is clearly the most convenient method to express objective visual loss.

Considering the traditional role of GPs, it is perhaps surprising to see that their rate of counselling is significantly lower than referrals made by optometrists (97 vs 18\%, $P=0.0001$ ). This low rate of documented counselling by GPs is of concern. It is possible that GPs are counselling their patients, but do not document in the referral that the patient has been counselled and remains keen for surgery. However, this is unlikely to explain the large difference observed, as unlike optometrists, GPs have not had prior explicit training highlighting the importance of such counselling and probably all do not have ready access to the necessary written information relating to cataract surgery that optometrists can provide. This low rate of counselling by GPs may partly explain the statistically significant, lower operative rate for the GP pathway.

One reason why referrals initiated by GPs are more likely to comment on a patient's personal circumstance (32 vs 3\%, $P=0.0001$ ) may be because the GP as the family doctor is in a more appropriate situation to discuss this with the patient. Furthermore, there is little opportunity on the direct optometric pathway form to comment on personal circumstances, which is unfortunate as such information can be useful to the HES for prioritising needy patients on their waiting list for cataract surgery. However, even for patients referred by their GP, the rate of referrals including information on personal circumstances is still low (32\%), which perhaps can be explained by only a small proportion of patients having significant personal circumstances that the referrer felt the HES should be aware of. This could be clarified by referrers being requested to state the absence of significant personal circumstances, such as effect on employment, driving, ability to care for dependents, or risk of falling.

Referrals initiated by the optometrists contained less information relating to past medical history (95 vs 68\%, $P=0.0001$ ) and drug history (94 vs 69\%, $P=0.0009$ ), which could be secondary to a lack of information completed by the GP. One way to overcome this would be for the HES to then send the form back to the GP and not accept the referral until all parts of the form had been completed.

Information on drug history is important, because certain drugs can make cataract surgery more difficult. There is evidence that anticoagulants and antiplatelets can complicate both cataract surgery and anaesthesia, and different centres manage patients in different ways. ${ }^{8}$ A recent large-scale study has demonstrated that systemic $\alpha$-blockers are also associated with increased operative complications. ${ }^{9}$ As patients referred via the GP pathway are more likely to have their drug history reported, it would be expected that more patients with a significant drug history are identified and managed appropriately (risks explained at consent, being listed for senior surgeon, and perhaps drug being omitted prior to surgery). However, as no significant complications were noted in the study, and postoperative visual acuity was the same for each pathway, it is not possible to conclude from this study that these different rates of reported drug history altered outcome.

There is no statistical difference in the percentage of cases where optometrists or GPs provide information relating to subjective visual loss (74 vs $77 \%, P=0.8342)$. However, GPs are more likely to qualify this. For example, GP referrals, relative to optometric referrals, are 
more likely to comment on visual loss/dim vision/ blurring (73 vs 2\%, $P=0.0001$ ) or difficulty with reading (15 vs $0 \%, P=0.0029$ ). This is probably because the direct optometric form simply has a tick box answer to subjective loss of vision, providing the optometrist little opportunity to further comment on this item.

There is no section on the direct optometric form that allows explicit comments relating to rapid reduction in visual acuity, anisometropia, or acute blepharitis (clinical factors deemed important by the College of Optometrists). Although there is a space on the form titled, 'other clinically relevant information', none of the optometrists and only $2 \%$ of the GPs commented on these factors.

Nearly all patients who were listed for surgery subsequently received surgery. Operative rates for direct optometric referrals were higher than traditional GP referrals, and this difference was statistically significant (87 vs 69\%, confidence interval for odds ratio 1.19-7.47, $P=0.0284$ ). More patients from the traditional GP pathway were not listed, because the cataract was found to have no effect on their lifestyle (12 from GP pathway, 4 from direct pathway), or because the patient declined surgery (four from GP pathway, two from direct pathway), or for other reasons (three from GP pathway, two from direct pathway). However, these differences in individual reasons why patients were not listed for surgery did not reach statistical significance, when compared between the pathways. A larger study with greater statistical power would be required to establish the reasons why patients are not listed for surgery, and whether or not there is any true discrepancy in reasons why patients are not listed between the two pathways.

Despite the difference in pathway referral characteristics and operative rates, the best-corrected visual acuities before and after surgery were the same for both pathways.

This study is not able to comment on how useful patients found the counselling provided by optometrists, as we did not collect these data. It is not possible to attribute observed differences between the referral pathways specifically to the counselling itself, only to the pathway as a whole. Patient satisfaction with counselling provided by optometrists has been investigated previously, suggesting good levels of patient satisfaction with optometrist assessment, information provision, and communication. ${ }^{6}$

It would be interesting to establish the relative costs of these pathways, from the perspective of the HES, optometrists, and primary care trust. Further studies with such cost/benefit analysis being the primary aim would be required to establish this.

These data have demonstrated that, in Avon and South Gloucestershire, referrals initiated by optometrists are better at providing information relating to objective visual loss and counselling, whereas referrals initiated by GPs are better at providing a more personalised referral including medical and drug information. The operative rate was slightly higher for referrals initiated by the optometrists. Despite these discrepancies in referral and operative rates, the pre- and postoperative visual acuities were the same for both pathways.

As a response to this information, a single referral form for use by optometrists and GPs has been developed locally with a view to improving referral information received across both pathways.

\section{Acknowledgements}

We thank Rosemary Greenwood, United Bristol Healthcare Trust, for her help relating to statistical analysis.

\section{References}

1 NHS Executive. Action on cataracts: good practice guidance. Department of Health, London, 2000.

2 Framework for the optometric co-management of patients with cataracts. College of Optometrists 2003.

3 Framework for optometric referrals. College of Optometrists 2005.

4 National Eye Care Services Steering Group. First report of the National Eye Care Services Steering Group. Department of Health, London, 2004.

5 Sharp J, Moriarty A, Warburton T, Dixon J. Cataract assessment and direct referral. Stockport optometrists take the initiative. Optom Today 2003; 25: 16-17.

6 Newsom W, Gibbons H, Flanagan D, Hingorani M. Direct referral of cataracts in Huntingdon. Sharing good practice. Optom Today 2005; 22: 21-24.

7 Lash SC, Prendiville CP, Samson A, Lewis K, Munneke R, Parkin BT. Optometrist referrals for cataract and 'Action on Cataracts' guidelines: are optometrists following them and are they effective? Ophthalmic Physiol Opt 2006; 26: 464-467.

8 Davies BR. Combined aspirin and clopidogrel in cataract surgical patients: a new risk factor for ocular haemorrhage? Br J Ophthalmol 2004; 88: 1226-1227.

9 Chung AKK, Johnston RL, Benzimra J, Jaycock P, Narendran $\mathrm{N}$, Chang BYP, et al., the EPR User Group. The Cataract National Dataset electronic multi-centre audit of 55,567 operations: alpha-blockers, operative complications and visual outcome. Eye 2007; (submitted). 